\title{
ON PROJECTIONS IN POWER SERIES SPACES AND THE EXISTENCE OF BASES
}

\author{
JÔRG KRONE
}

(Communicated by William J. Davis)

\begin{abstract}
Mityagin posed the problem, whether complemented subspaces of nuclear infinite type power series spaces have a basis. A related more general question was asked by Pełczyński. It is well known for a complemented subspace $E$ of a nuclear infinite type power series space, that its diametral dimension can be represented by $\Delta E=\Delta \Lambda_{\infty}(\alpha)$ for a suitable sequence $\alpha$ with $\alpha_{j} \geq$ $\ln (j+1)$. In this article we prove the existence of a basis for $E$ in case that $\alpha_{j} \geq j$ and $\sup \frac{\alpha_{2 j}}{\alpha_{j}}<\infty$.
\end{abstract}

It was shown by Mityagin, that complemented subspaces of nuclear finite type power series spaces always have a basis, and he asked, whether the same is valid for infinite type (cf. $[3,4,5])$. Dubinsky and Vogt [2] obtained a positive solution for some nuclear power series spaces $\Lambda_{\infty}(\alpha)$, namely they assumed that the set of all finite limit points of $\left\{\frac{\alpha_{i}}{\alpha_{j}}: i, j \in \mathbf{N}\right\}$ is bounded. Results for some other special cases are stated below. Pelczyński [6] posed the more general problem, whether complemented subspaces of nuclear Fréchet spaces with basis again have a basis. Both problems are open up to now.

Every complemented subspace $E$ of a nuclear infinite type power series space has the same diametral dimension as a power series space $\Lambda_{\infty}(\alpha)$ for a suitable sequence $\alpha$ with $\alpha_{j} \geq \ln (j+1)$ (cf. Terzioglu [8]). Considering isomorphisms between spaces of analytic functions Zaharjuta [15] conjectured that $E$ has a basis for stable $\alpha$ (this means $\sup _{j} \frac{\alpha_{2 j}}{\alpha_{j}}<\infty$ ). This will be proved in the present note in case $\alpha_{j} \geq j$. Other positive solutions have been obtained if one of the three following assumptions is satisfied:

(1) $\Lambda_{\infty}(\alpha)$ is a complemented subspace of $E$ (cf. Vogt [10]).

(2) There is a tame projection onto $E$ (cf. Dubinsky/Vogt [2]).

(3) $E$ is isomorphic to $E \oplus E$ (cf. Wagner [14], see also [11]).

The present proof uses result no. 1 of Vogt [10]. The main tool is the construction of a basis and of a projection in $E$ by a permuted Gram-Schmidt orthonormalization.

Received by the editors April 28, 1987 and, in revised form, March 22, 1988.

1980 Mathematics Subject Classification (1985 Revision). Primary 46A45; Secondary 46E10. 
Notation. See also Dubinsky [1] and Terzioglu [7]. Let $\alpha$ denote an exponens sequence, this is a nondecreasing, unbounded sequence of positive numbers.

A power series space of infinite type is defined by

$$
\Lambda_{\infty}(\alpha):=\left\{x=\left(x_{i}\right)_{i \notin \mathbf{N}}:\|x\|_{k}:=\sum_{i=1}^{\infty}\left|x^{i}\right| \exp \left(k \alpha_{i}\right)<\infty \quad \text { all } k \notin \mathbf{N}\right\} .
$$

For $\alpha_{j}=\ln (j+1)$ is $\Lambda_{\infty}(\alpha)=s$ and for $\alpha_{j}=(j)^{1 / n}$ is $\Lambda_{\infty}(\alpha) \cong H\left(\mathbf{C}^{n}\right)$, especially $\Lambda_{\infty}(\mathbf{N}) \cong H(\mathbf{C})$.

Let $E$ be a Fréchet space with a fundamental system of seminorms \|\|$_{k}$, $k \notin \mathbf{N}$. The corresponding neighbourhoods of zero are denoted by $U_{k}$.

$$
\begin{gathered}
E \text { has } D N \text { iff } \exists p \forall k \exists m, C: x \notin E\|x\|_{k}^{2} \leq C\|x\|_{m}\|x\|_{p} . \\
E \text { has } \Omega \text { iff } \forall p \exists k \forall m \exists n, C: r U_{k}<C r^{n} U_{m}+C / r U_{p} .
\end{gathered}
$$

In the nuclear case the condition $D N$ is characteristic for the subspaces of $s$ (cf. Vogt [9]) and $\Omega$ for the quotients of $s$ (cf. Vogt/Wagner [12]).

The Diametral Dimension $\Delta E$ is defined by

$$
\Delta E:=\left\{\left(x_{i}\right)_{i \notin \mathbf{N}}: \forall k \exists m: \lim _{i}\left|x_{i}\right| d_{i}\left(U_{m}, U_{k}\right)=0\right\},
$$

where the Kolmogorov diameters are $d_{i}(V, U):=\inf \{d>0: V \subset d U+L$ with $L \subset E$ and its dimension $\leq i\}$. The main part of this note is the proof of the following proposition.

Proposition 1. For an exponens sequence $\alpha$ with $\sup _{i} \frac{\alpha_{2 i}}{\alpha_{i}}<\infty$ and $\alpha_{i} \geq i$ for all $i \notin \mathbf{N}$ every quotient $E$ of $\Lambda_{\infty}(\alpha)$ with $D N$ and with $\Delta E=\Delta \Lambda_{\infty}(\alpha)$ has a complemented subspace isomorphic to $\Lambda_{\infty}(\alpha)$.

Proof. Let $U_{k} \supset U_{k+\mu}, k \notin \mathbf{N}$, Hilbert balls and a fundamental system of neighbourhoods of zero in $E$ so that for all $k$ there are $m, C$ with $\|x\|_{k}^{2} \leq$ $C\|x\|_{m}\|x\|_{0}$ for all $x \notin E$.

The surjection from $\Lambda_{\infty}(\alpha)$ onto $E$ is denoted by $q$ and there is a basis $\left(e_{i}\right)_{i \notin \mathbf{N}}$ in $\Lambda_{\infty}(\alpha)$ with $\left\|q\left(e_{i}\right)\right\|_{0} \leq \exp \left(-\alpha_{i}\right)$. For every $k \notin \mathbf{N}$ there is an $m_{k}$ so that $\left\|q\left(e_{i}\right)\right\|_{k} \leq \exp \left(m_{k} \alpha_{i}\right)$ for all $i$.

First we want to show the following lemma.

Lemma 1. There is a number $N$ such that for every $i \notin \mathbf{N}$ and every operator $T \notin L\left(\Lambda_{\infty}(\alpha), E\right)$ with $\operatorname{dim}(\operatorname{range}(T))<i$ we find $a j \notin \mathbf{N}$ with $\left\|q\left(e_{j}\right)-T\left(e_{j}\right)\right\|_{0} \geq \exp \left(-N \alpha_{i}\right)$.

Proof. If the lemma were false there would be a sequence $\left(i_{n}\right)_{n \notin \mathbf{N}}$ and $T_{n} \notin L\left(\Lambda_{\infty}(\alpha), E\right)$ with $\operatorname{dim}\left(\operatorname{range}\left(T_{n}\right)\right)<i_{n}$ so that for all $j \notin \mathbf{N}$ $\left\|q\left(e_{j}\right)-T\left(e_{j}\right)\right\|_{0} \leq \exp \left(-n \alpha_{i_{n}}\right)$. Hence there is an $m_{0}$ with $U_{m_{0}}<\exp \left(-n \alpha_{i_{n}}\right) U_{0}$ $+\operatorname{range}\left(T_{n}\right)$. 
Since $E$ has property $D N$, for every $k$ there are $m, C$ with $d_{i_{n}}\left(U_{m}, U_{k}\right) \leq$ $d_{i_{n}}\left(U_{m_{0}}, U_{0}\right)$ (e.g. see Terzioglu [8] condition (7)) and with $\exp \left(n \alpha_{i_{n}}\right) d_{i_{n}}$ $\left(U_{m}, U_{k}\right) \leq C$. Since $\Delta E=\Delta \Lambda_{\infty}(\alpha)$, there is a $K$ with $n \alpha_{i_{n}} \leq K \alpha_{i_{n}}$ for all $n \notin \mathbf{N}$, hence the lemma must be true.

Lemma 2. There is an orthonormal system $\left(f_{i}\right)_{i \notin \mathbf{N}}$ in $\left(E,\|\|_{0}\right)$ and a sequence of positive numbers $\left(\mu_{i}\right)_{i \notin \mathbf{N}}$, such that for all $j \notin \mathbf{N}$

(a) $\left\|\left(i d-P_{j-1}\right) q e_{i}\right\|_{0} \leq \frac{1}{\mu_{j}}$ for all $i$ and for $P_{\nu}(x):=\sum_{k=1}^{\nu}\left(f_{k}, x\right)_{0} f_{k}$.

(b) $\mu_{j} \leq \exp \left(N \alpha_{j}\right)$

(c) $\left\|f_{j}\right\|_{k} \leq \mu_{j} 2^{j} \exp \left(m_{k} N \alpha_{j}\right)$ for all $k$.

Proof. We want to prove Lemma 2 by induction over $j$. Since

$$
0 \leq \lim _{i}\left\|\left(i d-P_{j-1}\right) q e_{i}\right\|_{0} \leq \lim _{i}\left\|q e_{i}\right\|_{0}=0,
$$

there is an $n_{j}$ with $\left\|\left(i d-P_{j-1}\right) q e_{n_{j}}\right\|_{0}=\sup _{i}\left\|\left(i d-P_{j-1}\right) q e_{i}\right\|_{0}$. Let

$$
\mu_{j}:=\frac{1}{\left\|\left(i d-P_{j-1}\right) q e_{n_{j}}\right\|_{0}} \quad \text { and } \quad f_{j}:=\mu_{j}\left(i d-P_{j-1}\right) q e_{n_{j}} .
$$

Then $f_{j}$ is orthogonal to $f_{1}, f_{2}, \ldots, f_{j-1},\left\|f_{j}\right\|_{0}=1$ and (a) is valid. Since $\exp \left(-N \alpha_{j}\right) \leq \sup _{i}\left\|\left(i d-P_{j-1}\right) q e_{i}\right\|_{0}=\frac{1}{\mu_{j}}$, (b) is satisfied.

We use the following estimates to show (c):

$$
\begin{aligned}
\left\|\left(i d-P_{j-1}\right) q e_{n_{j}}\right\|_{k} & \leq\left\|q\left(e_{n_{j}}\right)\right\|_{k}+\sum_{\nu=1}^{j-1}\left|\left(f_{\nu}, q e_{n_{j}}\right) 0\right|\left\|f_{\nu}\right\|_{k} \\
& \leq\left\|q\left(e_{n_{j}}\right)\right\|_{k}+\sum_{\nu=1}^{j-1}\left|\left(f_{\nu},\left(i d-P_{\nu-1}\right) q e_{n_{j}}\right)_{0}\right|\left\|f_{\nu}\right\|_{k} \\
& \leq \exp \left(m_{k} \alpha_{n_{j}}\right)+\sum_{\nu=1}^{j-1} \frac{1}{\mu_{\nu}} \mu_{\nu} 2^{\nu} \exp \left(m_{k} N \alpha_{\nu}\right) \\
& \leq \exp \left(m_{k} \alpha_{n_{j}}\right)+\exp \left(m_{k} N \alpha_{j}\right)\left(2^{j}-1\right) .
\end{aligned}
$$

Hence it is sufficient to show $\alpha_{n_{j}} \leq N \alpha_{j}$. But this fact follows from $\exp \left(-N \alpha_{j}\right)$ $\leq\left\|\left(i d-P_{j-1}\right) q e_{n_{j}}\right\|_{0} \leq\left\|q e_{n_{j}}\right\|_{0} \leq \exp \left(-\alpha_{n_{j}}\right)$.

Lemma 3. There is a projection $P$ in $E$ with $\operatorname{range}(P)$ isomorphic to $\Lambda_{\infty}(\alpha)$. Proof. Let $n_{k}:=\left(m_{k} N+N+2\right) C$ with $\sup _{j} \frac{\alpha_{2 j}}{\alpha_{j}} \leq C$. Now we construct an orthonormal sequence $\left(g_{j}\right)_{j \notin \mathbf{N}}$ in $E$ with respect to $(,)_{0}$ so that $g_{j}$ is orthogonal to $g_{1}, g_{2}, \ldots, g_{j-1}, q e_{1}, q e_{2}, \ldots, q e_{j}$ and $g_{j}=\sum_{i=1}^{2 j}\left|x_{i}\right| f_{i}$ with $\sum_{j=1}^{2 j}\left|x_{i}\right|^{2}=1$. Then

$$
\begin{aligned}
\left\|g_{j}\right\|_{k} & \leq \sum_{i=1}^{2 j}\left|x_{i}\right|\left\|f_{i}\right\|_{k} \leq 2 j \max _{i \leq 2 j} \mu_{i} 2^{i} \exp \left(m_{k} N \alpha_{i}\right) \\
& \leq \exp \left(n_{k} \alpha_{j}\right)
\end{aligned}
$$


and

$$
\begin{aligned}
\left\|\left(g_{j}, q\left(\sum_{i=1}^{\infty} x_{i} e_{i}\right)\right)_{0} g_{j}\right\|_{k} & \leq \sum_{i=j+1}^{\infty}\left|x_{i}\left(g_{j}, q e_{i}\right)_{0}\right|\left\|g_{j}\right\|_{k} \\
& \leq \sum_{i=j+1}^{\infty}\left|x_{i}\right| \exp \left(-\alpha_{j}\right) \exp \left(n_{k} \alpha_{j}\right) \\
& \leq \exp \left(-\alpha_{j}\right) \sum_{i=1}^{\infty}\left|x_{i}\right| \exp \left(n_{k} \alpha_{i}\right) \leq \exp \left(-\alpha_{j}\right)\|x\|_{n_{k}} .
\end{aligned}
$$

Hence $P(x):=\sum_{j=1}^{\infty}\left(g_{j}, x\right)_{0} g_{j}$ defines a projection in $E$ and $\left(g_{j}\right)_{j \notin \mathbf{N}}$ is a basis in the range of $P$. For $a_{j, k}:=\left\|g_{j}\right\|_{k}$ is $\lambda(A) \cong \operatorname{range}(P)$ a Köthe sequence space with $D N, \Omega$ and $\Delta \lambda(A) \supset \Delta E=\Delta \Lambda_{\infty}(\alpha)$. Since $\left\|g_{j}\right\|_{0}=1$ and $\left\|g_{j}\right\|_{k} \leq \exp \left(n_{k} \alpha_{j}\right)$ we obtain $\Delta \lambda(A) \subset \Delta \Lambda_{\infty}(\alpha)$, hence $\Lambda_{\infty}(\alpha) \cong \lambda(A) \cong$ range $(P)$.

Now we apply Proposition 1 to show our main result.

Theorem 1. For an exponens sequence with $\sup _{j} \frac{\alpha_{2 j}}{\alpha_{j}}<\infty$ and $\alpha_{j} \geq j$ for all $j \notin \mathbf{N}$ a Fréchet space $E$ is isomorphic to $\Lambda_{\infty}(\alpha)$ if and only if $E$ has the properties $D N, \Omega$ and $\Delta E=\Delta \Lambda_{\infty}(\alpha)$.

Corollary. A Fréchet space $E$ is isomorphic to $H(\mathbf{C})$ if and only if $E$ has the properties $D N, \Omega$ and $\Delta E=\Delta \Lambda_{\infty}(\mathbf{N})$.

Proof. If $E$ has the properties $D N, \Omega$ and $\Delta E=\Delta \Lambda_{\infty}(\alpha)$, then due to Vogt/Wagner [13] $E$ is isomorphic to a complemented subspace of $\Lambda_{\infty}(\alpha)$. On the other hand Proposition 1 shows that $\Lambda_{\infty}(\alpha)$ is isomorphic to a complemented subspace of $E$. Hence Vogt [10] yields that $E$ is isomorphic to $\Lambda_{\infty}(\alpha)$ (cf. result no. 1 stated in the introduction).

In the proof of the theorem we can use instead of Proposition 1 the following modification:

Proposition 1*. Let $\alpha$ be an exponens sequence with $\sup _{j} \frac{\alpha_{2 j}}{\alpha_{j}}<\infty$ and $\alpha_{j} \geq j$ for all $j \notin \mathbf{N}$, and let $E$ be a subspace of $\Lambda_{\infty}(\alpha)$. If $\Delta E=\Delta \Lambda_{\infty}(\alpha)$ and if $E$ is isomorphic to a quotient of $\Lambda_{\infty}(\alpha)$, then there is a complemented subspace of $\Lambda_{\infty}(\alpha)$, which is both contained in $E$ and isomorphic to $\Lambda_{\infty}(\alpha)$.

Proof. In the proof of Proposition 1, Lemma 3 we construct a $g_{j}$ orthogonal to $g_{1}, g_{2}, \ldots, g_{j-1}, q e_{1}, q e_{2}, \ldots, q e_{j}$. For a proof of Proposition $1^{*}$ we merely have to exchange this by $g_{j}$ orthogonal to $g_{1}, g_{2}, \ldots, g_{j-1}, e_{1}, e_{2}, \ldots, e_{j}$.

Finally we want to point out that it is an interesting problem to prove Proposition 1 or $1^{*}$ for some other exponens sequences. Especially we want to know, whether it is sufficient to assume $\alpha_{j} \geq \ln (j+1)$ instead of $\alpha_{j} \geq j$. This was stated without proof by Zaharjuta [15], for his work we need in particular the cases $\alpha_{j}=(j)^{1 / n}$. Most important are sequences $\alpha_{j}=a^{j}$, because then 
Proposition $1^{*}$ would solve the above mentioned prob'lems of Mityagin and Pełczyński.

Theorem 2. If the claim made in Proposition $1^{*}$ were true for $\alpha_{j}=a^{j}$ with an $a>1$, then there would be a complemented subspace of $s$ without basis.

Proof. $\omega$ is the set of all sequences endowed with the seminorms $\left\|\left(x_{i}\right)_{i \in \mathbf{N}}\right\|_{k}:=$ $\max _{i \leq k}\left|x_{i}\right|$ for all $k \in \mathbf{N}$. For every $a>1$ and $\alpha_{j}:=a^{j}$ there is a surjection $T$ from $\Lambda_{\infty}(\alpha)$ onto $\omega$, so that the kernel of $T$ called $K$ has the properties $\Omega$ and $\Delta K=\Delta \Lambda_{\infty}(\alpha)$. This result is proved by Vogt [8]. Now we want to consider the case that every complemented subspace of $s$ has a basis, hence that $K$ is isomorphic to $\Lambda_{\infty}(\alpha)$. If Proposition $1^{*}$ were true for this $\alpha$, then there would be a projection $P$ in $\Lambda_{\infty}(\alpha)$, so that the range of $P$ is contained in $K$ and isomorphic to $\Lambda_{\infty}(\alpha)$. The first condition implies that $T$ restricted to $F=$ : $\operatorname{range}(i d-P)$ is still a surjection onto $\omega$. Since $F \oplus \Lambda_{\infty}(\alpha) \cong$ $F \oplus \operatorname{range}(P)=\Lambda_{\infty}(\alpha)$, this yields a contradiction, because here $\alpha_{j}=a^{j}$ with $a>1$, so that $\Lambda_{\infty}(\alpha)$ is not isomorphic to $\Lambda_{\infty}(\alpha) \oplus \Lambda_{\infty}(\beta)$ for every exponens sequence $\beta$.

\section{ACKNOWLEDGMENT}

I wish to thank A. Aytuna, R. Meise, D. Vogt and M. Wagner for several useful discussions and suggestions concerning this paper.

\section{REFERENCES}

1. E. Dubinsky, The structure of nuclear Fréchet spaces, Lecture Notes in Math., vol. 720, Springer-Verlag, 1979.

2. E. Dubinsky and D. Vogt, Basis in complemented subspaces of power series spaces, Bull. Acad. Polon. Sci. 34 (1986), 65-67.

3. B. S. Mityagin, Sur l'equivalence des bases inconditionelles dans les echelles de Hilbert, C. R. Acad. Sci. Paris 269 (1969), 426-428.

4. __, Equivalence of bases in Hilbert scales, Studia Math. 37 (1971), 111-137. (Russian)

5. B. S. Mityagin and G. Henkin, Linear problems in complex analysis, Uspekhi. Mat. Nauk 26(4) (1971), 93-152.

6. A. Pełczyński, Problem 37, Studia Math. 38 (1970), 476.

7. T. Terzioğlu, Die diametrale dimension von lokalkonvexen Räumen, Collect. Math. 20 (1969), 49-99.

8. $\ldots$, On the diametral dimension of some classes of F-spaces, J. of Karadeniz U. 8 (1985), $1-13$.

9. D. Vogt, Charakterisierung der Unterrräume von s, Math. Z. 155 (1977), 109-117.

10. __ Ein isomorphiesatz für potenzreihenräume, Arch. Math. 38 (1982), 540-548.

11. __ Kernels of Eidelheit matrices and related topics, doga 10.1 (1986), 232-256.

12. D. Vogt and M. J. Wagner, Charakterisierung der quotienten von $s$ und eine vermutung von martineau, Studia Math. 67 (1980), 225-240. 
13. __ Unterräume und quotienten der nuklearen, stabilen potnezreihenräume von unendlichem typ, Studia Math. 70 (1981), 63-80.

14. M. J. Wagner, Stable complemented subspaces of $(s)$ have a basis, Seminar Lecture, AG Funktionalanalysis Düsseldorf/Wuppertal, 1985.

15. V. D. Zaharjuta, Isomorphism of spaces of analytic functions, Soviet Math. Dokl. 22 (1980), 631-634.

Bergische Universität-Gesamthochschule WUPPERTAL, Fachbereich Mathlematik, Gaubstr. 20, D-5600 Wuppertal 1, Federal Republic of Germany 